\title{
Initial-Boundary Value Problems for First Order Hyperbolic Systems
}

\author{
By
}

\section{Kunihiko Kajitani*}

\section{$\S 1$ Introduction}

In this paper we shall obtain energy inequalities and existence theorem of the solutions to hyperbolic mixed problems with constant coefficients.

Let us consider the following mixed problems in a quarter space:

$$
\begin{cases}\frac{\partial}{\partial t} u(t)=L[u(t)]+f(t) & \text { in } t>0, x>0, y \in \mathbf{R}^{n} \\ \left.u_{t}\right|_{=0}=g & \text { in } x>0, y \in \mathbf{R}^{n} \\ \left.B u\right|_{x=0}=0 & \text { in } t>0, y \in \mathbf{R}^{n}\end{cases}
$$

where

$$
L=A \frac{\partial}{\partial x}+\sum_{j=1}^{n} B_{j} \frac{\partial}{\partial y_{j}}+C(x, y)
$$

$A$ and $B_{j}(j=1, \ldots, n)$ are $N \times N$ constant matrices and $C(x, y)$ is sufficiently smooth, and $B$ is $l \times N$ matrix.

Moreover assumptions on $(L, B)$ are as the followings:

A.I) $L$ is strictly hyperbolic, that is, $A \xi+\sum B_{j} \eta_{j}$ has real distinct eigen values for $\xi \in \mathbf{R}^{1}, \eta=\left(\eta_{1}, \ldots, \eta_{n}\right) \in \mathbf{R}^{n},(\xi, \eta) \neq 0$, and $A$ is non singular.

A.II) The multiplicity of the real eigen values of $A^{-1}\left(\sigma+\sum B_{j} \eta_{j}\right)$ is not greater than two for $\sigma \in \mathbf{R}^{1}, \eta \in \mathbf{R}^{n},(\sigma, \eta) \neq 0$.

Put $M(\tau, \eta)=A^{-1}\left(\tau+i \sum B_{j} \eta_{j}\right), \operatorname{Re} \tau>0, \eta \in \mathbf{R}^{n}$. We denote by $E_{-}(\tau, \eta)$ Received January 10, 1971.

Conmunicated by S. Matsuura.

* Department of Applied Mathematics and Physics, Faculty of Engineering, Kyoto University, Yoshida, Kyoto, Japan. 
the span of all the ordinary and generalized eigen vectors of $M(\tau, \eta)$ corresponding to it's eigen values with negative real parts. Hyperbolicity of $L$ implies that $\operatorname{dim} E_{-}(\tau, \eta)$ is constant for $\operatorname{Re} \tau>0, \eta \in \mathbf{R}^{n}$ and it is equal to the number of negative eigenvalues of $A$. Let $\mathbf{r}_{j}(\tau, \eta)(j=1, \ldots, k)$ be basis of $E_{-}(\tau, \eta)$, where $k=\operatorname{dim} E_{-}(\tau, \eta)$, and $H(\tau, \eta)=\left(\mathbf{r}_{1}, \ldots, \mathbf{r}_{k}\right)$ is $N \times k$ matrix. Then $l \times k$ matrix $B \cdot H(\tau, \eta)$ varies continuously in $\operatorname{Re} \tau$ $\geqq 0, \eta \in \mathbf{R}^{n},(\tau, \eta) \neq 0$.

A.III) $B$ satisfies the uniform Lopatinski's condition, that is, $l=k$ and $\operatorname{det}(B \cdot H(\tau, \eta))$ is not zero for $\operatorname{Re} \tau \geqq 0, \eta \in \mathbf{R}^{n},(\tau, \eta) \neq 0$.

Denote a definition domain of $L$ by $\mathscr{D}(L)=\left\{u, L u \in L^{2}\left(\mathbf{R}_{+}^{n+1}\right),\left.B u\right|_{x=0}\right.$ $=0\}$.

Our main results are the following:

Theorem 1.1. There exist positive constant $\mu_{0}$ and $c$ such that, for any $\tau$ with $\operatorname{Re} \tau>\mu_{0}(\tau-L)$ is a one to one and onto map from $\mathscr{D}(L)$ to $L^{2}\left(\mathbf{R}_{+}^{n+1}\right)$ and for any positive integer $m$ holds

$$
\left\|(\tau-L)^{-m}\right\|_{L^{2}\left(\mathbf{R}_{+}^{n+1}\right) \leqq c} \frac{1}{\left(\operatorname{Re} \tau-\mu_{0}\right)^{m}} .
$$

Theorem 1.2. For any $f(t)$ in $\mathscr{E}_{t}^{1}\left(L^{2}\left(\mathbf{R}_{+}^{n+1}\right)\right)^{1)}$ and $\frac{\partial}{\partial y} f(t)(j=1$, $\ldots, n)$ in $\mathscr{E}_{t}^{0}\left(L^{2}\left(\mathbf{R}_{+}^{n+1}\right)\right.$ ) and for initial data $g$ in $H^{1}\left(\mathbf{R}_{+}^{n+1}\right) \cap \mathscr{D}(L)$, there exists a unique solution of (1.1) which is in $\mathscr{E}_{t}^{1}\left(L^{2}\left(\mathbf{R}_{+}^{n+1}\right)\right) \cap \mathscr{E}_{t}^{0}\left(H^{1}\left(\mathbf{R}_{+}^{n+1}\right)\right)$ $\cap \mathscr{D}(L)$, such that

$$
\begin{aligned}
& \left\|\frac{\partial}{\partial t} u(t)\right\|^{2}+\|u(t)\|_{1}^{2} \\
& \leqq c(T)\left\{\|g\|_{1}^{2}+\int_{0}^{t}\left(\|f(s)\|^{2}+\left\|\frac{\partial}{\partial s} f(s)\right\|^{2}+\sum_{j=1}^{n}\left\|\frac{\partial}{\partial y_{j}} f(s)\right\|^{2}\right) d s\right\}
\end{aligned}
$$

for $t \in[0, T]$, where $\|\cdot\|\left(\|\cdot\|_{1}\right)$ is a norm of $L^{2}\left(\mathbf{R}_{+}^{n+1}\right)\left(H^{1}\left(\mathbf{R}_{+}^{n+1}\right)\right)$.

\section{§ 2. Stationary Problems}

In this section we shall show the existence of the resolvent for the 1) $f(t) \in \mathscr{E}_{l}^{p}(E)$ means that $f(t)$ is $p$ times continuously differentiable with values in $E(p=0,1, \ldots)$. 
operator $L$. Let $L_{0}$ denote the principal part of $L$ and $\mathscr{D}\left(L_{0}\right)$ it's definition domain. It is noted that $\mathscr{D}\left(L_{0}\right)$ is equal to $\mathscr{D}(L)$.

Lemma 2.1. Under A.I), A.II) and A.III), there exists a positive constant $\delta$ depending only on $L_{0}$ such that

$$
\left\|\left(\tau-L_{0}\right) u\right\| \geqq \delta \operatorname{Re} \tau\|u\|
$$

for all $u \in H^{1}\left(\mathbf{R}_{+}^{n+1}\right)$ and for $\tau, \operatorname{Re} \tau>0$.

Proofs of Lemma 3.1 may be founded in $([8],[9],[15],[16])$. It should be remarked that the condition A.II can be removed for $L_{0}$ with real constant coefficients. (Cf. [16]. [9].)

Lemma 2.2 (Hersh [6]). Suppose A.I) and A.III). Then the inage $\left(=-L_{0}\right) \mathscr{D}\left(L_{0}\right)$ is dense in $L^{2}\left(R_{+}^{n+1}\right)$ for any complex number $\tau$ with $\operatorname{Re} \tau$ $>0$.

This is the special case of R. Hersh [6]. In fact he proved this lemma under the conditions which are the hyperbolicity on $L_{0}$ (not necessarily strictly) and $\operatorname{det}(B \cdot H(\tau, \eta)) \neq 0$ for $(\tau, \eta)$ with $\operatorname{Re} \tau>0$.

Lemma $2.1,2.2$ and 2.5 imply immediately the following

Lemma 2.3. Suppose A.I), A.II) and A. III). Then the operator ( $\left.\tau-L_{0}\right)$ applies one to one and onto from $\mathscr{D}\left(L_{0}\right)$ to $L^{2}\left(\mathbf{R}_{+}^{n+1}\right)$ for $\operatorname{Re} \tau>0$.

Next we consider the operator $L$. Noting $L=L_{0}+C$, we have

Lemma 2.4. Suppose A.I), A.II) and A.III). Then the operator $L$ applies one to one and onto from $\mathscr{D}(L)$ to $L^{2}\left(\mathbf{R}_{+}^{n+1}\right)$ for any $\tau$ with $\operatorname{Re} \tau$ $>\beta$ and satisfies

$$
\|(\tau-L)\| \geqq \delta(\operatorname{Re} \tau-\beta)\|u\|
$$

for all $u \in \mathscr{D}(L)$ and $\operatorname{Re} \tau>\beta$, where $\delta$ and $\beta$ are positive constants independent of $\tau$.

Proof. It follows from (2.1) that

$$
\operatorname{li}^{\prime}(\tau-L) u\|\geqq\|\left(\tau-L_{0}\right) u\|-\| C u \|
$$




$$
\geqq \delta \operatorname{Re} \tau\|u\|-\|C u\| \text {. }
$$

Therefore, $\beta=\sup |C(x, y)| / \delta$ implies (2.2). Next we must prove that $(\tau-L) \mathscr{D}(L)$ is dense in $L^{2}\left(\mathbf{R}_{+}^{n+1}\right)$. If it is not so, there exists $v \neq 0$ in $L^{2}\left(\mathbf{R}_{+}^{n+1}\right)$ such that

$$
((\tau-L) u, v)=0
$$

for all $u \in \mathscr{D}(L)$, where $($,$) is the inner product of L^{2}\left(\mathbf{R}_{+}^{n+1}\right)$. Noting $\mathscr{D}(L)=\mathscr{D}\left(L_{0}\right)$, by Lemma 2.3 we have $u \in \mathscr{D}(L)$ such that

$$
\left(\tau-L_{0}\right) u=v \text {. }
$$

This, (2.3) and (2.1) imply

$$
\begin{aligned}
\|v\|^{2} & =(C u, v) \\
& \leqq \frac{\beta}{\operatorname{Re} \tau}\|v\|^{2} .
\end{aligned}
$$

When $\operatorname{Re} \tau>\beta$, (2.4) contradicts $v \neq 0$.

Q.E.D.

Remark. When a positive constant $\delta$ in Lemma 2.4 is larger than one, we have

$$
\left\|(\tau-L)^{-1}\right\|_{L^{2}\left(\mathbf{R}_{+}^{n+1}\right)} \leqq \frac{1}{\operatorname{Re} \tau-\beta} \text { for } \operatorname{Re} \tau>\beta
$$

which implies immediately Theorem 2.1. But in general we can not expect so.

Put $v=(\tau-L)^{-1} g(\operatorname{Re} \tau>\beta)$. We need a lemma to verify the regurality of $v$.

Lemma 2.5 (Lax-Phillips [10]). The definition domain $\mathscr{D}(L)$ is identified with the graph norm closure of

$$
\mathrm{D}_{\mathrm{L}}=\left\{u \in H^{1}\left(\mathbf{R}_{+}^{n+1}\right) ;\left.B u\right|_{x=0}=0\right\}
$$

Lemma 2.6. When $g$ belongs to $H^{1}\left(\mathbf{R}_{+}^{n+1}\right)$ and $\mathscr{D}(L), v=(\tau-L)^{-1} g$ is in $H^{1}\left(\mathbf{R}_{+}^{n+1}\right)$ and satisfies

$$
\|v\|_{1} \leqq c(\operatorname{Re} \tau)\|g\|_{1},
$$


where $c(\operatorname{Re} \tau)$ depends only on $\operatorname{Re} \tau$ and $L$.

Proof. From (2.2) we have

$$
{ }_{1}^{\prime} v\|\leqq c(\operatorname{Re} \tau)\| g \|
$$

Further the fact that $g$ in $\mathscr{Z}(L)$ and the relation $\tau v=(\tau-L)^{-1} \tau g=(\tau-$ $L)^{-1} L g+g$ imply that

$$
\begin{aligned}
\|\tau v\| & \leqq c(\operatorname{Re} \tau)(\|g\|+\|L g\|) \\
& \leqq c(\operatorname{Re} \tau)\|g\|_{1} .
\end{aligned}
$$

If we can prove that $\frac{\partial}{\partial y_{k}} v(k=1, \ldots, n)$ are in $\mathscr{D}(L)$, our assertion will be proved. In fact, since $v$ satisfies the equation

$$
\left(\tau-A \frac{\partial}{\partial x}-\sum B_{j} \frac{\partial}{\partial y_{j}}-C\right) v=g
$$

$\frac{\partial}{\partial y_{k}} v$ satisfies

$$
\left(\tau-A \frac{\partial}{\partial x}-\sum B_{j} \frac{\partial}{\partial y_{j}}\right) \frac{\partial}{\partial y_{k}} v=\left(\frac{\partial}{\partial y_{k}} C\right) \cdot v+\frac{\partial}{\partial y_{k}} g
$$

in distribution sense. If $\frac{\partial}{\partial y_{k}} v$ belongs to $\mathscr{D}(L)$, we have

$$
(\tau-L) \frac{\partial}{\partial y_{k}} v=\left(\frac{\partial}{\partial y_{k}} C\right) v+\frac{\partial}{\partial y_{k}} g
$$

which implies

$$
\begin{aligned}
\left\|\frac{\partial}{\partial y_{k}} v\right\| & \leqq c(\operatorname{Re} \tau)\left(\left\|\left(\frac{\partial}{\partial y_{k}} C\right) v\right\|+\left\|\frac{\partial}{\partial y_{k}} g\right\|\right) \\
& \leqq c(\operatorname{Re} \tau)\|g\|_{1} .
\end{aligned}
$$

Noting that $A$ is non singular, we can obtain (2.5) from (2.7), (2.8) and (2.9).

We prove that $\frac{\partial}{\partial y_{k}} v(k=1, \ldots, n)$ are in $\mathscr{D}(L)$. Let $\rho(y)$ be any infinitely differential non negative function with compact support and total mass one; 


$$
\int_{\mathbf{R}^{n}} \rho(y) d y=1
$$

The function $\rho_{\varepsilon}(y)$ and $v_{\varepsilon}$ are defined for positive $\varepsilon$, as

$$
\rho_{\varepsilon}=\varepsilon^{-n} \rho\left(\frac{y}{\varepsilon}\right)
$$

and

$$
v_{\varepsilon}=\rho_{\varepsilon} * v=\int_{\mathbf{R}^{n}} \rho_{\varepsilon}\left(y-y^{\prime}\right) v\left(x, y^{\prime}\right) d y^{\prime}
$$

respectively.

According to Lemma 2.5, it is easily seen that $\frac{\partial}{\partial y_{k}} v_{\varepsilon}$ is in the predomain $\mathrm{D}_{\mathrm{L}}$ for $\varepsilon>0$. From (2.8) we obtain the following relation

$$
(\tau-L) \frac{\partial}{\partial y_{k}} v_{\varepsilon}=f_{\varepsilon} \rho_{\varepsilon} * \frac{\partial}{\partial y_{k}} g+\rho_{\varepsilon}^{*}\left(\left(\frac{\partial}{\partial y_{k}} C\right) \cdot v\right)
$$

where $f_{\varepsilon}=C\left(\rho_{\varepsilon}^{*} \frac{\partial}{\partial y_{k}} v\right)-\rho_{\varepsilon} *\left(C \cdot \frac{\partial}{\partial y_{k}} v\right)$, which, as well known, belongs to $L^{2}\left(\mathbf{R}_{+}^{n+1}\right)$ and satisfies

$$
\left\|f_{\varepsilon}\right\| \leqq c\|v\|
$$

and

$$
\lim _{\varepsilon \rightarrow 0} f_{\varepsilon}=0 \quad \text { in } L^{2}\left(\mathbf{R}_{+}^{n+1}\right)
$$

From (2.10) and (2.2) it follows that

$$
\begin{gathered}
\left\|\frac{\partial}{\partial y_{k}} v_{\varepsilon}-\frac{\partial}{\partial y_{k}} v_{\varepsilon^{\prime}}\right\| \leqq c(\operatorname{Re} \tau)\left(\left\|f_{\varepsilon}-f_{\varepsilon^{\prime}}\right\|+\left\|\left(\rho_{\varepsilon}-\rho_{\varepsilon^{\prime}}\right) * \frac{\partial}{\partial y_{k}} g\right\|\right. \\
+\left\|\left(\rho_{\varepsilon}-\rho_{\varepsilon^{\prime}}\right) *\left(\frac{\partial}{\partial y_{k}} C\right) v\right\|
\end{gathered}
$$

which right side tends to zero limiting $\varepsilon, \varepsilon^{\prime} \rightarrow 0$. Hence $\left\{\frac{\partial}{\partial y_{k}} v_{\varepsilon}\right\}$ is Cauchy sequence and has a limit $(\varepsilon \rightarrow 0)$ which is in $L^{2}\left(\mathbf{R}_{+}^{n+1}\right)$. It is obvious that the limit is equal to $\frac{\partial}{\partial y_{k}} v$. And also from (2.10) it follows that $L\left(\frac{\partial}{\partial y_{k}}\right.$ $\left.v_{\varepsilon}\right)$ tends to $L\left(\frac{\partial}{\partial y_{k}} v\right)$ in $L^{2}\left(\mathbf{R}_{+}^{n+1}\right)$ for $\varepsilon \rightarrow 0$. This shows that $\frac{\partial}{\partial y_{k}} v$ 
belongs to $\mathscr{D}(L)$.

Corollary. When $g$ is in $\mathscr{D}\left(\mathbf{R}_{+}^{n+1}\right), v=(\tau-L)^{-1} g$ belongs to $H^{s}\left(\mathbf{R}_{+}^{n+1}\right)$ and satisfies

$$
\|v\|_{s} \leqq c(\operatorname{Re} \tau, s)\|g\|_{s}
$$

for any posilive integer $s$, where $\mathscr{D}\left(\mathbf{R}_{+}^{n+1}\right)$ consists of infinitely differentiable functions with compact support.

Now we consider a lemma needed later on. Let us put $\tau=\mu+i \sigma$ $(\mu, \sigma$ real $)$ and $v(\tau)=(\tau-L)^{-1} g$. Then we have

Lemma 2.7. Suppose A.I), A.II) and A.III), then there exists a positive constant $c(\mu)$ depending only on $\mu$ and $L$ such that

$$
\begin{aligned}
& \int_{-\infty}^{\infty}\|v(\tau)\|^{2} d \sigma \leqq c(\mu)\|g\|^{2} \\
& \int_{-\infty}^{\infty}\left\langle v(\tau)>^{2} d \sigma \leqq c(\mu)\|g\|^{2}\right.
\end{aligned}
$$

for all $g \in H^{1}\left(\mathbf{R}_{+}^{n+1}\right) \cap \mathscr{D}(L)$ and $\mu>\beta$, where

$$
\left\langle v(\tau)>^{2}=\int_{\mathbf{R}^{n}}|v(\tau, 0, y)|^{2} d y\right.
$$

and $v(\tau, 0, y)$ means a trace of $v(\tau)$.

The proofs of Lemma 2.7 can be seen in [8] and [16]. When $g$ is in $\mathscr{D}\left(\mathbf{R}_{+}^{n+1}\right)$, from (2.13) we have, by the same way as the proof of the corollary of Lemma 2.6,

Corollary. When $g$ is in $\mathscr{D}\left(\mathbf{R}_{+}^{n+1}\right)$, it holds that

$$
\int_{-\infty}^{\infty}\|v(\tau)\|_{s}^{2} d \sigma \leqq c(\mu, s)\|g\|_{s}^{2}
$$

for any positive integer $s$.

We define the operator $S_{t}(t>0)$ for $g \in \mathscr{D}\left(\mathbf{R}_{+}^{n+1}\right)$ as

$$
S_{t} g=\frac{1}{2 \pi} \int_{-\infty}^{\infty} e^{\tau t}(\tau-L)^{-1} g d \sigma .
$$

The finiteness of the right side of (2.15) is assured by (2.13). In fact, considering $\frac{1}{2 \pi} \int_{-\infty}^{\infty} e^{\tau t} \tau^{-1} d \sigma=1$, we can write 


$$
S_{t} g=g+\frac{1}{2 \pi} \int_{-\infty}^{\infty} \frac{e^{\tau t}}{\tau}(\tau-L)^{-1} \operatorname{Lg} d \sigma
$$

from which we can estimate roughly by (2.13),

$$
\left\|S_{t} g\right\| \leqq\|g\|+c(\mu) e^{\mu t}|| \operatorname{Lg} \| \quad(\mu>\beta) .
$$

Further it should be remarked that $S_{t} g$ is independent of $\mu$. In fact we can easily see that $v(\tau)=(\tau-L)^{-1} g$ is an analytic function of $\tau$, considering the estimate (2.6).

In the estimate (2.17) we can remove the term $\|\mathrm{Lg}\|$ from the right side, that is,

Lemma 2.8. Suppose A.I), A.II) and A.III). There exist positive constants $c$ and $\mu_{0}$ such that

$$
\left\|S_{t} g\right\| \leqq c e^{\mu_{0} t}\|g\| \quad(\iota>0)
$$

for all $g \in \mathscr{D}\left(\mathbf{R}_{+}^{n+1}\right)$.

The proof of Lemma 2.8 will be given in the next section.

Now we can show that Theorem 1.1 follows from Lemma 2.8.

Proof of Theorem 1.1. Let $\lambda$ be a complex number with $\operatorname{Re} \lambda>\mu_{0}$. Then we can show

$$
(\lambda-L)^{-1} g=\int_{0}^{\infty} e^{-\lambda t} S_{t} g d t .
$$

In fact, according to (2.13) and (2.16), we obtain by Fubini's theorem,

$$
\begin{aligned}
\int_{0}^{\infty} e^{-\lambda t} S_{t} g d t & =\frac{1}{2 \pi} \int_{-\infty}^{\infty}\left(\int_{0}^{\infty} e^{-(\lambda-\tau) t} d t\right)(\tau-L)^{-1} g d \sigma \\
& =\frac{1}{2 \pi} \int_{-\infty}^{\infty}(\lambda-\tau)^{-1}(\tau-L)^{-1} g d \sigma
\end{aligned}
$$

where $\tau=\mu+i \sigma\left(\mu_{0}<\mu<\operatorname{Re} \lambda\right)$. Considering that $(\tau-L)^{-1} g$ is analytic in $\tau$ with $\operatorname{Re} \tau>\mu_{0}$, we obtain the relation (2.19). Then $m$ times differentiation of (2.19) with respect to $\lambda$ leads us to a relation

$$
(-1)^{m}(\lambda-L)^{-1} g=\int_{0}^{\infty}(-t)^{m-1} e^{-\lambda t} S_{t} g d t,
$$

from which we can derive by using (2.18)

$$
\left\|(\lambda-L)^{-m} g\right\| \leqq \int_{0}^{\infty} t^{m-1} e^{-\operatorname{Re} \lambda t}\left\|S_{t} g\right\| d t
$$




$$
\leqq c \cdot\left(\operatorname{Re} \lambda-\mu_{0}\right)^{-m}\|g\|
$$

for $\operatorname{Re} \lambda>\mu_{0}$. This shows (1.2) because $\mathscr{D}\left(\mathbf{R}_{+}^{n+1}\right)$ is dense in $L^{2}\left(\mathbf{R}_{+}^{n+1}\right)$. The proof is complete.

\section{§ 3. Energy Inequality}

In this section we shall derive an energy inequality of the mixed problem (1.1) with $f(t)=0$, and using it we shall prove Lemma 2.8 stated in the previous section. We should point out that the method of proof given here may be related to the results of [5].

We now consider a preliminary lemma well known, which proof is seen in $[4]$.

Lemma 3.1. Suppose that $a(\xi, \eta)=A \xi+\sum_{j=1}^{n} B_{j} \eta_{j}$ has only real and distinct eigenvalues for all real $(\xi, \eta) \neq 0$. Then there exists a matrix $\gamma(\xi$, $\eta)$ having following properties.

1) $\gamma(\xi, \eta)$ is a hermitian positive definite matrix with homogeneous of degree zero for all real $(\xi, \eta) \neq 0$.

2) $\gamma(\xi, \eta) a(\xi, \eta)$ is hermitian.

3) $\gamma(\xi, 0)$ is a constant hermitian matrix ( $\left.\equiv \gamma_{0}\right)$.

4) $\gamma(\lambda, \eta)$ is an analytic function matrix of $\lambda$ in the strip

$$
|\operatorname{Im} \lambda| \leqq \varepsilon|\eta|, \quad \text { for } \eta \neq 0,
$$

where $\varepsilon$ is a positive constant, $(\xi, \eta)=\left(\xi, \eta_{1}, \ldots, \eta_{n}\right)$ and $|\eta|=\left(\eta_{1}^{2}+\cdots+\eta_{n}^{2}\right)^{\frac{1}{2}}$.

We define a singular integral operator $R$ as

$$
R \varphi=\check{\gamma} * \varphi=\int_{\mathbf{R}^{n+1}} \check{\gamma}\left(x-x^{\prime}, y-y^{\prime}\right) \varphi\left(x^{\prime}, y^{\prime}\right) d x^{\prime} d y^{\prime}
$$

for $\varphi \in \mathscr{D}\left(\mathbf{R}^{n+1}\right)$, where $\breve{\gamma}(x, y)$ stands for a inverse Fourier image of $\gamma(\xi, \eta)$. It follows from 1 ) of Lemma 3.1 that $R$ is a symmetric positive operator, that is,

$$
(R \varphi, \varphi) \geqq \delta\|\varphi\|^{2}
$$

for $\varphi \in L^{2}\left(\mathbf{R}^{n+1}\right)$, where $\delta$ is a positive constant.

Let $\varphi$ be in $H^{1}\left(\mathbf{R}_{+}^{n+1}\right) \cap C^{1}\left(\mathbf{R}_{+}^{n+1}\right)$ and $\tilde{\varphi}$ denote an extension of $\varphi$ which is equal to zero in $x<0$, that is, 


$$
\tilde{\varphi}(x, y)= \begin{cases}\varphi(x, y) & \text { for } x \geqq 0 \\ 0 & \text { for } x<0 .\end{cases}
$$

We decompose $\gamma(\xi, \eta)=\gamma(\xi, 0)+\gamma(\xi, \eta)-\gamma(\xi, 0)=\gamma_{0}+\gamma_{1}(\xi, \eta)$. Then it follows from Lemma 3.1 that $\gamma_{0}$ is constant and that $\gamma_{1}(\xi, \eta)$ has an estimate

$$
\left|\gamma_{1}(\xi, \eta)\right| \leqq c \cdot \frac{|\eta|}{|\xi|}
$$

for $\xi \neq 0, \eta \neq 0$. Let $R_{1}$ be a singular integral operator with it's symbol $\gamma_{1}(\xi, \eta)$. Then we have

Lemma 3.2. The following relation holds

$$
\left(R_{1} \frac{\partial}{\partial x} \varphi, \psi\right)=-\left(\varphi, \frac{\partial}{\partial x}\left(R_{1} \tilde{\psi}\right)\right)-<\varphi, R_{1} \tilde{\psi}>
$$

for any $\varphi, \psi$ in $H^{1}\left(\mathbf{R}_{+}^{n+1}\right)$, where $($,$) means the inner product of$ $L^{2}\left(\mathbf{R}_{+}^{n+1}\right)$ and

$$
\left\langle\varphi, \psi>=\int_{\mathbf{R}^{n}} \varphi(0, y) \cdot \psi(0, y) d y\right.
$$

Proof. When $\varphi$ belongs to $H^{1}\left(\mathbf{R}_{+}^{n+1}\right)$, it follows from (3.3) that $\frac{\partial}{\partial x} R_{1} \tilde{\varphi}$ is in $L^{2}\left(\mathbf{R}^{n+1}\right)$. In fact we have by virtue of Parseval's equality,

$$
\begin{aligned}
\int_{\mathbf{R}^{n+1}}\left|\frac{\partial}{\partial x} R \tilde{\varphi}\right|^{2} d x d y & =\int_{\mathbf{R}^{n+1}}\left|i \xi \gamma_{1}(\xi, \eta) \hat{\tilde{\varphi}}(\xi, \eta)\right|^{2} d \xi d \eta \\
& \leqq c \cdot \int_{\mathbf{R}^{n+1}}|\eta|^{2}|\hat{\tilde{\varphi}}(\xi, \eta)|^{2} d \xi d \eta \\
& \leqq c \cdot\|\varphi\|_{1}^{2}
\end{aligned}
$$

where $\hat{\tilde{\varphi}}(\xi, \eta)$ stands for a Fourier image of $\tilde{\varphi}(x, y)$ and $\|\cdot\|_{1}$ means the norm of $H^{1}\left(\mathbf{R}_{+}^{n+1}\right)$. Hence we can regard $R_{1} \tilde{\varphi}$ as a continuous function $\left(R_{1} \tilde{\varphi}\right)(x)$ from $(-\infty, \infty)$ to $L^{2}\left(\mathbf{R}^{n}\right)$ and also obtain the relation (3.4) integrating by part. Moreover noting $\left(R_{1} \tilde{\varphi}\right)(x)$ in $\mathscr{E}_{x}^{0}\left(L^{2}\left(\mathbf{R}^{n}\right)\right)$ for $x \in \mathbf{R}^{1}$, we have

$$
<R_{1} \tilde{\varphi}, \psi>=\int_{\mathbf{R}^{n}}\left(\int_{-\infty}^{\infty} \gamma_{1}(\xi, \eta) \hat{\tilde{\varphi}}(\xi, \eta) d \xi\right) \cdot \hat{\psi}(0, \eta) d \eta
$$

where $\hat{\psi}(0, \eta)$ is a Fourier image of $\phi(0, y)$ with respect to $y$. Q. E. D. 
Corollary. We have the following relation

$$
\begin{aligned}
& (R \widetilde{L u}, u)+(R \tilde{u}, L u) \\
& =2 \operatorname{Re}(R \widetilde{C u}, u)-2 \operatorname{Re}<R_{1} A \tilde{u}, u>-<\gamma_{0} A u, u>
\end{aligned}
$$

for any $u \in H^{1}\left(\mathbf{R}_{+}^{n+1}\right)$, where $L=A \frac{\partial}{\partial x}+\sum_{j=1}^{n} B_{j} \frac{\partial}{\partial y_{j}}+C$.

We now consider the following evolution equation

$$
\left\{\begin{array}{l}
\frac{d}{d t} u(t)=L u(t) \\
u(0)=g .
\end{array}\right.
$$

Then we are to derive an energy inequality for a solution of the equation (3.7).

Theorem 3.1. Suppose that a solution $u(t)$ of (3.7) belongs to the pre-domain $\mathrm{D}_{\mathrm{L}}$ and $\mathscr{E}_{t}^{1}\left(L^{2}\left(\mathbf{R}_{+}^{n+1}\right)\right)$, and further that $u(t), \frac{\partial}{\partial t} u(t)$ and $\frac{\partial}{\partial y_{j}} u(t)(j=1, \ldots, n)$ are in $L_{\mu_{0}}^{2}\left((0, \infty) ; L^{2}\left(\mathbf{R}_{+}^{n+1}\right)\right) .{ }^{1)}$ Then under A.I), A.II) and A.III) there exists a positive constant $c\left(\mu_{0}\right)$ depending only on $\mu_{0}$ and $L$ such that

$$
\|u(t)\| \leqq c\left(\mu_{0}\right) e^{\mu_{0} t}\|g\|
$$

for $t \in[0, \infty)$.

Proof. Let $R$ be the singular integral operator defined in (3.1). Then noting $R \widetilde{u(t)} \in \mathscr{E}_{t}^{1}\left(L^{2}\left(\mathbf{R}_{+}^{n+1}\right)\right)$, we have

$$
(R \tilde{u}(t), u(t))=(R \tilde{u}(0), u(0))+\int_{0}^{t} \frac{d}{d t}(R \tilde{u}(t), u(t)) d t,
$$

from which we can derive by means of the corollary of Lemma 3.2,

$$
\begin{aligned}
(R \tilde{u}(t), u(t)) & =(R \check{g}, g)+2 \int_{0}^{t}\{\operatorname{Re}(R \widetilde{C u(t)}, u(t)) \\
& \left.-\frac{1}{2}<\gamma_{0} A u(t), u(t)>-\operatorname{Re}<R_{1} A \tilde{u}(t) \cdot u(t)>\right\} d t .
\end{aligned}
$$

1) $L_{\mu_{0}}^{2}\left((0, \infty) ; L^{2}\left(R_{+}^{n+1}\right)\right)$ consists of all functions $u(t)$ such that

$$
\int_{0}^{\infty} e^{-2 \mu_{0} t}\|u(t)\|^{2} d t<\infty
$$


Let us put $\tau=\mu_{0}+i \sigma$ and define $v(\tau, x, y)$ and $\hat{v}(\tau, \xi, \eta)$ as

$$
v(\tau, x, y)=\int_{0}^{\infty} e^{-\tau t} u(t, x, y) d t
$$

and

$$
\hat{v}(\tau, \tilde{\varsigma}, \eta)=\int_{\mathbf{R}^{n+1}} e^{-x \xi-i y \eta} \tilde{v}(\tau, x, y) d x d y
$$

respectively. It is noted that hold

$$
\int_{-\infty}^{\infty}\|v(\tau)\|^{2} d \sigma=\int_{0}^{\infty} e^{-2 \mu_{0} t}\|u(t)\|^{2} d t
$$

and

$$
\int_{-\infty}^{\infty}\langle v(\tau)\rangle^{2} d \sigma=\int_{0}^{\infty} e^{-2 \mu_{0} t}\left\langle u(t)>^{2} d t .\right.
$$

From (3.10) we can estimate

$$
\begin{aligned}
& (\widetilde{R u(t)}, u(t)) \leqq c \cdot\left(\|g\|^{2}+\int_{0}^{t}\left(\|u(t)\|^{2}+<R_{1} \widetilde{A u(t)}>^{2}+>u(t)>^{2}\right) d t\right) \\
& \leqq c \cdot\left(\|g\|^{2}+e^{2 \mu_{0} t} \int_{-a}^{\infty}\left(\|v(\tau)\|^{2}+<R_{1} A v(\tau)>^{2}\right.\right. \\
& \left.\left.+\langle v(\tau)\rangle^{2}\right) d \sigma\right)
\end{aligned}
$$

Our purpose is to prove the following inequality

$$
\int_{-\infty}^{\infty}<R_{1} A v(\tau)>^{2} d \sigma \leqq c\left(\|g\|^{2}+\int_{-\infty}^{\infty}\left(\|v(\tau)\|^{2}+<v(\tau)>^{2}\right) d \sigma\right) .
$$

Combining (3.12) with Lemma 2.7 in the previous section, we can prove our theorem. In fact, $v(\tau)$ belongs to the definition domain $\mathscr{D}(L)$ because $u(t)$ is in the pre-domain $\mathrm{D}_{\mathrm{L}}$ and $v(\tau)$ is a limit of $v_{j}(\tau)$ in $\mathscr{D}(L)(j \rightarrow \infty)$ such that

$$
v_{j}(\tau)=\int_{0}^{j} e^{-\tau t} u(t) d t
$$

And since $v(\tau)$ satisfies the equation

$$
(\tau-L) v(\tau)=g
$$

by Lemma 2.7 we can estimate

$$
\int_{-\infty}^{\infty}\left(\|v(\tau)\|^{2}+\left\langle v(\tau)>^{2}\right) d \sigma \leqq c \cdot\|g\|^{2},\right.
$$

from which and (3.12) we obtain 


$$
(\widetilde{R u(t)} \cdot u(t)) \leqq c e^{\mu_{0} t}\|g\|^{2}
$$

This and (3.2) imply (3.8).

We must now prove the inequality (3.12). Considering the representation (3.5), we have

$$
\int_{-\infty}^{\infty}<R_{1} A \widetilde{A v(\tau)}>^{2} d \sigma=\int_{\mathbf{R}^{n+1}}|F(\tau, \eta)|^{2} d \sigma d \eta
$$

where

$$
F(\tau, \eta)=\frac{1}{2 \pi} \int_{-\infty}^{\infty} \gamma_{1}(\xi, \eta) A \hat{v}(\tau, \xi, \eta) d \xi
$$

To prove (3.12), we shall show that there exists a positive constant $K_{0}$ depending only on $\mu_{0}$ and $L$ such that

$$
\begin{aligned}
\int_{-\infty}^{\infty}|F(\tau, \eta)|^{2} d \sigma & \leqq c\left\{\int_{-\infty}^{\infty}\left(\int_{-\infty}^{\infty}|\hat{v}(\tau, \xi, \eta)|^{2} d \xi+|\bar{v}(\tau, 0, \eta)|^{2}\right) d \sigma\right. \\
& \left.+\int_{-\infty}^{\infty}|\hat{g}(\xi, \eta)|^{2} d \xi\right\}
\end{aligned}
$$

for $|\eta| \geqq K_{0}$, where $\hat{g}(\xi, \eta)$ is a Fourier image of $\tilde{g}(x, y)$ and $\bar{v}(\tau, 0, \eta)$ a Fourier image of $v(\tau, 0, y)$ with respect to $y$.

When $|\eta| \leq K_{0}$, we have

$$
|F(\tau, \eta)| \leqq \int_{|\xi| \leqq 1}\left|\gamma_{1}(\xi, \eta) A \hat{v}(\tau, \xi, \eta)\right| d \xi+\int_{|\xi| \geqq 1}\left|\gamma_{1}(\xi, \eta) A \hat{v}(\tau, \xi, \eta)\right| d \xi
$$

and from (3.3) we have obtained by Schwarz's inequality

$$
\begin{aligned}
\int_{|\eta| \leqq K_{0}}|F(\tau, \eta)|^{2} d \sigma d \eta & \leqq c \int_{-\infty}^{\infty}\left(|| R_{1} A \tilde{v}(\tau) \|^{2}+|v(\tau)|^{2}\right) d \sigma \\
& \leqq c \int_{-\infty}^{\infty} \mid v(\tau \mid)^{2} d \sigma .
\end{aligned}
$$

We turn now to deriving the inequality (3.15). To do so, we consider the representation (3.14) for $F(\tau, \eta)$. According to Lemma 3.1, $\gamma_{1}(\lambda, \eta)$ is analytic with respect to $\lambda$ in the strip $|\operatorname{Im} \lambda| \leqq \varepsilon|\eta|$, and $\hat{v}(\tau, \lambda, \eta)$ is an analytic function of $\lambda$ in $\operatorname{Im} \lambda<0$ because it is the Fourier transform of $\tilde{v}(\tau, x, y)$ which is zero for $x<0$. Therefore we may shift the line of integration in (3.14) into the complex plane, that is,

$$
F(\tau, \eta)=\frac{1}{2 \pi} \int_{\operatorname{Im} \lambda=-m} \gamma_{1}(\lambda, \eta) A \hat{v}(\tau, \lambda, \eta) d \lambda
$$


where $0 \leqq m \leqq \varepsilon|\eta|$.

Since $v(\tau, x, y)$ satisfies the differential equation

$$
\left(\tau-A \frac{\partial}{\partial x}-\sum_{j=1}^{n} B_{j} \frac{\partial}{\partial y_{j}}-C\right) v(\tau, x, y)=g(x, y)
$$

taking the Fourier transform of it with respect to $(x, y)$, we obtain the following relation for $\hat{v}(\tau, \lambda, \eta)$

$$
(\tau-i a(\lambda, \eta)) \hat{v}(\tau, \lambda, \eta)=A \bar{v}(\tau, 0, \eta)+\hat{f}(\tau, \lambda, \eta)+\hat{g}(\lambda, \eta),
$$

where $\hat{f}(\tau, \lambda, \eta)$ stands for a Fourier image of $C(x, y) v(\tau, x, y)$ with respect to $(x, y)$ and

$$
a(\lambda, \eta)=A \lambda+\sum_{j=1}^{n} B_{j} \eta_{j}
$$

Let us note that there exists a positive constant $K_{1}$ such that $M(\tau$, $\eta)=A^{-1}\left(\tau-i \sum B_{j} \eta_{j}\right)$ has distinct eigenvalues for $|\tau| \geqq K_{1}|\eta|$. Because it follows from the condition A.I) that eigenvalues of $A$ are distinct. To estimate $\hat{v}(\tau, \lambda, \eta)$ on $\operatorname{Im} \lambda=-m$ by use of the relation (3.18), we must give two different arguments when $|\tau| \leqq K_{1}|\eta|$ and $|\tau| \geqq K_{1}|\eta|$.

To estimate $\hat{v}(\tau, \lambda, \eta)$ for $|\tau| \leqq K_{1}|\eta|$, we shall need the following lemma (cf. K.O. Friedrichs and P.D. Lax [5], Lemma 4.1);

Lemma 3.3. Suppose that $|\tau| \leqq K_{1}|\eta|$. Then there exists a $m=$ $m(\eta)$ in the range

$$
\frac{1}{2} \varepsilon|\eta| \leqq m \leqq \varepsilon|\eta|
$$

such that

$$
\mid(\tau-i a(\lambda, \eta))^{-1} \leqq \frac{\text { const. }}{|\lambda|}
$$

for all $\lambda$ with $\operatorname{Im} \lambda=m(\eta)$.

Proof. Using (3.19) and the assumed fact that $A$ is non singular, we can write

$$
\left.(\tau-i a(\lambda, \eta))=i \lambda A\left\{A^{-1} \lambda^{-1}\left(\frac{\tau}{i}-\sum B_{j} \lambda_{j}\right)\right)-1\right\}
$$


which shows that $(\tau-i a(\lambda, \eta))$ is invertible for $|\tau| \leqq K_{1}|\eta|$ and $|\lambda| \geqq$ $\frac{1}{2}\left|A^{-1}\right|\left(K_{1}+\sum\left|B_{j}\right|\right)|\eta|=K_{2}|\eta|$ and $\left|(\tau-i a(\lambda, \eta))^{-1}\right| \leqq \frac{2\left|A^{-1}\right|}{|\lambda|}$. Next we shall verify (3.21) for $|\lambda| \leqq K_{2}|\eta|$. Since (3.20) and (3.21) are homogeneous, we can normalize so that $|\eta|=1$. Let $\Gamma$ denote the set of $\lambda$ with $-\varepsilon \leqq \operatorname{Im} \lambda \leqq-\varepsilon / 2$ and $|\operatorname{Re} \lambda| \leqq K_{2}$. To each $\left(\tau_{0}, \eta_{0}\right)$ with $\left|\tau_{0}\right|^{2}+\left|\eta_{0}\right|^{2} \leqq K_{1}^{2}+1$ the number of the root $\lambda$ of $\left(\tau_{0}-i a\left(\lambda, \eta_{0}\right)\right)=0$ which belongs to $\Gamma$ is at most $l$. Since the roots $\lambda_{j}(\tau, \eta)$ of $(\tau-i a(\lambda, \eta))=0$ vary continuously, for $(\tau, \eta)$ in a sufficiently small neighbourhood $V\left(\tau_{0}\right.$, $\left.\eta_{0}\right)$ of $\left(\tau_{0}, \eta_{0}\right)$ with $\left|\tau_{0}\right|^{2}+\left|\eta_{0}\right|^{2} \leqq K_{1}^{2}+1$, it holds that

$$
\left|\lambda_{j}(\tau, \eta)-\lambda_{j}\left(\tau_{0}, \eta_{0}\right)\right| \leqq \frac{1}{6 l} \cdot \frac{\varepsilon}{2} \quad(j=1, \ldots, l) .
$$

Let $\Gamma\left(\tau_{0}, \eta_{0}\right)$ be the set of $\lambda=\xi-i m$ with $\varepsilon / 2 \leqq m \leqq \varepsilon$ which differs from any root $\lambda_{j}\left(\tau_{0}, \eta_{0}\right)(j=1, \ldots, l)$ at least by $\frac{1}{3 l} \cdot \frac{\varepsilon}{2}$. From (4.22) it follows that for $(\tau, \eta) \in V\left(\tau_{0}, \eta_{0}\right)$ and for $\lambda \in \Gamma\left(\tau_{0}, \eta_{0}\right)(\tau-i a(\lambda, \eta))^{-1}$ is bounded. Since the set $(\tau, \eta)$ with $|\tau|^{2}+|\eta|^{2} \leqq K_{1}^{2}+1$ is compact, it can be covered by finite open sets $V$. Hence the statement of Lemma 3.3 holds for any $(\tau, \eta)$ with $|\tau| \leqq K_{1}|\eta|$.

Q.E. D.

Solving (3.18) for $\hat{v}(\tau, \lambda . \eta)$, we can write

$$
\hat{\imath}(\tau, \lambda, \eta)=(\tau-i a(\lambda, \eta))^{-1}\{A v(\tau, 0, \eta)+f(\tau, \lambda, \eta)+g(\lambda, \eta)\}
$$

and from (3.21) we obtain the estimate

$$
|\hat{v}(\tau, \lambda, \eta)| \leqq \frac{c}{|\lambda|}\{|\dot{v}(\tau, 0, \eta)|+|\hat{f}(\tau, \lambda, \eta)|+\hat{g}(\lambda, \eta)\}
$$

Using this and (3.3) to estimate the integrand in (3.17), we can obtain

$$
\begin{aligned}
|F(\tau, \eta)|^{2} & \leqq c\left\{\int \frac{|\eta|}{|\lambda|^{2}}(|\bar{v}(\tau, 0, \eta)|+|\hat{f}(\tau, \lambda, \eta)|+|\hat{g}(\lambda, \eta)|) d \lambda\right\}^{2} \\
& \leqq c\left\{|\bar{v}(\tau, 0, \eta)|^{2}+\frac{1}{|\eta|} \int\left(|f(\tau, \lambda, \eta)|^{2}+|\hat{g}(\lambda, \eta)|^{2}\right) d \lambda\right\} .
\end{aligned}
$$

Integrating this with respect to $\sigma$, we get 


$$
\begin{aligned}
& \int_{|\tau| \leqq K_{1}|\eta|}|F(\tau, \eta)|^{2} d \sigma \\
& \leqq c\left\{\int_{-\infty}^{\infty}|\bar{i} \cdot(\tau, 0, \eta)|^{2} d \sigma+\iint|\hat{f}(\tau, \lambda, \eta)|^{2} d \lambda d \sigma+\int \mid \hat{g}(\lambda, \eta)^{2} d \lambda\right\} .
\end{aligned}
$$

We next estimate $F(\tau, \eta)$ for $|\tau| \leqq K_{1}|\eta|$. Then $M(\tau, \eta)=A^{-1}$ $\left(\tau-i \sum B_{j} \eta_{j}\right)$ having distinct eigenvalues, we have the expression as

$$
\begin{aligned}
(\tau-i a(\lambda, \eta))^{-1} & =A^{-1}(M(\tau, \eta)-i \lambda)^{-1} \\
& =\sum_{j=1}^{N} \frac{M_{j}(\tau, \eta)}{i \lambda-\lambda_{j}(\tau, \eta)}
\end{aligned}
$$

for $|\tau| \geqq K_{1}|\eta|$, where $\lambda_{j}(\tau, \eta)(j=1, \ldots, N)$ are eigenvalues of $M(\tau, \eta)$ and $M_{j}(\tau, \eta)$ are matrices homogeneous of degree zero in $(\tau, \eta)$. Let real parts of $\lambda_{j}(\tau, \eta)$ and it's imaginary parts denote by $\nu_{j}(\tau, \eta)$ and $\zeta_{j}(\tau, \eta)$ respectively. Then we need a lemma to estimate (3.26).

Lemma 3.4. Suppose that $|\tau| \geqq K_{1}|\eta|$ and $|\eta| \geqq K_{0}$, where $K_{0}$ is a sufficiently large constant. Then there exist positive constants $c\left(\mu_{0}\right)$ and $\delta$ which are depending only on $\mu(=\operatorname{Re} \tau)$ and $L$ such that

$$
\begin{aligned}
& \left|\nu_{j}(\tau, \eta)\right| \leqq c\left(\mu_{0}\right) \\
& \left|\frac{\partial}{\partial \sigma} \zeta_{j}(\tau, \eta)\right| \geqq \delta \quad(j=1, \ldots, N)
\end{aligned}
$$

where $\tau=\mu_{0}+i \sigma$.

Proof. For $|\tau| \geqq K_{1}|\eta| \lambda_{j}(\tau, \eta)(j=1, \ldots, N)$ are analytic functions of $(\tau, \eta)$. Hence we can write

$$
\begin{aligned}
\nu_{j}(\tau, \eta)-\nu_{j}(\tau, 0) & =\int_{0}^{1} \frac{d}{d \alpha} \nu_{j}(\tau, \alpha \eta) d \alpha \\
& =\int_{0}^{1} \sum_{k=1}^{n} \frac{\partial}{\partial \eta_{k}} \nu_{j}(\tau, \alpha \eta) \alpha d \alpha
\end{aligned}
$$

Let $a_{j}(j=1, \ldots, N)$ be eigenvalues of $A$. Then noting that $\lambda_{j}(\tau, 0)=$ $a_{j}^{-1} \tau$ and that $a_{j}(j=1, \ldots, N)$ are real, we have $\nu_{j}(\tau, 0)=a_{j}^{-1} \mu$. Since $\frac{\partial}{\partial \eta_{k}} \nu_{j}(\tau, \alpha \eta)$ are homogeneous of order zero, they are uniformly bounded for $|\tau| \geqq K_{1}|\eta|$ and $|\eta| \geqq K_{0}$. Thus the relation (3.29) implies (3.27). 
Since $\frac{\partial}{\partial \sigma} \zeta_{i}(\tau, \eta)$ are differentiable in $\eta$, we can write

$$
\frac{\partial}{\partial \sigma} \zeta_{j}(\tau, \eta)-\frac{\partial}{\partial \sigma} \zeta_{j}(\tau, 0)=\int_{0}^{1} \sum_{k=1}^{n} \frac{\partial}{\partial \eta_{k}}\left(\frac{\partial}{\partial \sigma} \zeta_{j}(\tau, \alpha \eta) \alpha d \alpha\right.
$$

Noting that $\frac{\partial}{\partial \sigma} \zeta_{j}(\tau, 0)=a_{j}^{-1}$ and that $\frac{\partial}{\partial \eta_{k}}\left(\frac{\partial}{\partial \sigma} \zeta_{j}(\tau, \alpha \eta)\right)$ are homogeneous of degree minus one, we can get

$$
\left|\frac{\partial}{\partial \eta_{k}}\left(\frac{\partial}{\partial \sigma} \zeta_{j}(\tau, \eta \sigma)\right)\right| \leqq \frac{1}{2 \mathrm{n}}\left|a_{j}^{-1}\right|
$$

for $|\tau| \geqq K_{2}|\eta|$ and $|\eta| \geqq K_{0}$, where $K_{0}$ is a sufficiently large positive constant. Thus (3.30) and (3.31) imply (3.28).

Q.E. D.

Further, let us note that considering (3.20) and (3.27) we get

$$
\left|m(\eta)-\nu_{j}(\tau, \eta)\right| \geqq \delta_{1}|\eta|
$$

for $|\tau| \geqq K_{1}|\eta|$ and $|\eta| \geqq K_{0}$, where $\delta_{1}$ is independent of $\sigma$ and $\eta$.

From (3.23) and (3.26) we obtain the estimate

$$
\begin{aligned}
|\hat{v}(\tau, \lambda, \eta)|^{2} \leqq & \sum_{j=1}^{N} \frac{c}{\left|\xi-\zeta_{j}(\tau, \eta)\right|^{2}+\left|m(\eta)-\nu_{j}(\tau, \eta)\right|^{2}}\left\{|\bar{v}(\tau, 0, \eta)|^{2}\right. \\
& \left.+|\hat{f}(\tau, \lambda, \eta)|^{2}+|\hat{g}(\lambda, \eta)|^{2}\right\}
\end{aligned}
$$

and from (3.3) we have by Schwarz's inequality

$$
\begin{aligned}
\left|F_{2}(\tau, \eta)\right|^{2} & \leqq c\left(\int \frac{|\eta|}{|\lambda|}|\hat{v}(\tau, \lambda, \eta)| d \lambda\right)^{2} \\
& \leqq c|\eta| \int|\hat{v}(\tau, \lambda, \eta)|^{2} d \lambda .
\end{aligned}
$$

Moreover, from (3.27) and (3.32) we can derive the following

$$
\begin{aligned}
& \int \frac{d \lambda}{\left|\lambda-\lambda_{j}(\tau, \eta)\right|^{2}} \leqq c \int_{-\infty}^{\infty} \frac{d \xi}{\left|\xi-\zeta_{j}(\tau, \eta)\right|_{j}+\left|m(\eta)-\nu_{j}(\tau, \eta)\right|^{2}} \\
& \leqq c \int_{-\infty}^{\infty} \frac{d \xi}{\xi^{2}+|\eta|^{2}} \\
&=c \frac{1}{|\eta|}
\end{aligned}
$$


for $|\eta| \geqq K_{0}$, and considering (3.28) we obtain by a change of the variable of integration

$$
\begin{aligned}
& \int_{\left|\tau i \geqq K_{1}\right| \eta \mid} \frac{d \sigma}{\left|\lambda-\lambda_{j}(\tau, \eta)\right|^{2}} \\
& \quad=\int_{|\tau| \geqq K_{1}|\eta|} \frac{d \sigma}{\left|\xi-\zeta_{j}(\tau, \eta)\right|^{2}+\left|m(\eta)-\nu_{j}(\tau, \eta)\right|^{2}}- \\
& \quad \leqq c \int_{-\infty}^{\infty} \frac{d \zeta}{\zeta^{2}+|\eta|^{2}} \quad\left(\zeta=\zeta_{j}(\tau, \eta)\right) \\
& \leqq c \frac{1}{|\eta|}
\end{aligned}
$$

for $|\eta| \geqq K_{0}$.

Using (3.35) and (3.36), we can derive from (3.33) and (3.34) the following

$$
\begin{gathered}
\int_{|\tau| \geqq K_{1}|\eta|}|F(\tau, \eta)|^{2} d \sigma \leqq c\left\{\int_{-\infty}^{\infty}|\bar{v}(\tau, 0, \eta)|^{2}+\int|\hat{f}(\tau, \lambda, \eta)|^{2} d \lambda\right) d \sigma \\
\left.+\int|\hat{g}(\lambda, \eta)|^{2} d \lambda\right\}
\end{gathered}
$$

Considering

$$
\int|\hat{f}(\tau, \lambda, \eta)|^{2} d \lambda \leqq c \int_{-\infty}^{\infty}|\hat{v}(\tau, \xi, \eta)|^{2} d \xi
$$

and

$$
\int|\hat{g}(\lambda, \eta)|^{2} d \lambda \leqq \int_{-\infty}^{\infty}|\hat{g}(\xi, \eta)|^{2} d \hat{\xi}
$$

we can derive (3.15) from (3.25) and (3.37). Integration of (3.15) with respect to $\eta$ and (3.16) imply our main inequality (3.12). This completes the proof of Theorem 3.1.

We can prove Lemma 2.8. If we can prove that $S_{t} g$ is a solution of (3.7) which satisfies the condition of Theorem 3.1, the lemma will be proved. We state as the followings:

Lemma 3.4. Let $g$ be in $\mathscr{D}\left(\mathbf{R}_{+}^{n+1}\right)$. Then $S_{t} g$ satisfies the following properties

1) $S_{t} g$ is in $\mathscr{D}(L)$ for $t>0$ and satisfies 


$$
\frac{d}{d t} S_{t} g=S_{t} L g=L S_{t} g
$$

2) $\lim _{t \rightarrow+0} S_{t} g=g \quad$ in $L^{2}\left(\mathbf{R}_{+}^{n_{+1}}\right)$.

Proof. We can write

$$
S_{t} g=g+\frac{1}{2 \pi} \int_{-\infty}^{\infty} \tau^{-1} e^{r t}(\tau-L)^{-1} L g d \sigma .
$$

Let us put

$$
u_{p}(\iota)=\frac{1}{2 \pi} \int_{-p}^{p} \tau^{-1} e^{\tau t}(\tau-L)^{-1} L g d \sigma,
$$

which clearly is in $\mathscr{D}(L)$ because $(\tau-L)^{-1} L g$ belongs to the pre-domain $\mathrm{D}_{\mathrm{L}}$ by virtue of the corollary of Lemma 2.6. Noting that $L(\tau-L)^{-1} L g$ $=(\tau-L)^{-1} L^{2} g$, we have

$$
L u_{p}(t)=\frac{1}{2 \pi} \int_{-p}^{p} \frac{e^{\tau t}}{\tau}(\tau-L)^{-1} L^{2} g d \sigma
$$

which converges in $L^{2}\left(\mathbf{R}_{+}^{n+1}\right)$ limiting $p \rightarrow \infty$ by virtue of Lemma 2.8. This implies that $S_{t} g$ in $\mathscr{D}(L)$ and that the following relation holds.

$$
\begin{aligned}
L S_{t} g & =L g+\frac{1}{2 \pi} \int_{-\infty}^{\infty} \frac{e^{\tau t}}{\tau}(\tau-L)^{-1} L^{2} g d \sigma \\
& =S_{t} L g .
\end{aligned}
$$

Noting that the residue calculus gives

$$
\frac{1}{2 \pi} \int_{-\infty}^{\infty} \frac{e^{\tau t}}{\tau^{2}} d \sigma=t \quad(t>0)
$$

we can write

$$
S_{t} g=g+t L g+\frac{1}{2 \pi} \int_{-\infty}^{\infty} \frac{e^{\tau t}}{\tau^{2}}(\tau-L)^{-1} L^{2} g d \sigma
$$

which right side belongs to $\mathscr{E}_{t}^{1}\left(L^{2}\left(\mathbf{R}_{+}^{n+1}\right)\right)$, hence we obtain

$$
\begin{aligned}
\frac{d}{d t} S_{t} g & =L g+\frac{1}{2 \pi} \int_{-\infty}^{\infty} \frac{e^{\tau t}}{\tau}(\tau-L) L^{2} g d \sigma \\
& =S_{t} L g
\end{aligned}
$$

which and (3.38) give the property 1). We prove 2). It follows from 
Lemma 3.7 that the second term in the right side of $(2.16)$ is in $\sigma_{t}^{0}\left(\mathbb{R}_{+}^{n+1}\right)$, hence it holds

$$
\lim _{t \rightarrow+0} S_{t} g=g+\frac{1}{2 \pi} \int_{-\infty}^{\infty} \frac{1}{\tau}(\tau-L)^{-1} \operatorname{Lg} d \sigma,
$$

which integration is independent of $\operatorname{Re} \tau$ because $(\tau-L)^{-1} \mathrm{Lg}$ is analytic in $\tau$. And considering Lemma 3.7, we can have

$$
\lim _{\operatorname{Re}^{\tau \rightarrow \infty}} \frac{1}{2 \pi} \int_{-\infty}^{\infty} \frac{1}{\tau}(\tau-L)^{-1} \operatorname{Lg} d \sigma=0
$$

which gives the property 2).

Q. E. D.

Proof of Lemma 2.8. Put $u(t)=S_{t}$ g. From Lemma 3.4 it follows that $u(t)$ and it's derivative in $t$ are in $\mathscr{E}_{t}^{0}\left(L^{2}\left(\mathbb{R}_{+}^{n+1}\right)\right)$ and $L_{\mu}^{2}\left((0, \infty) ; L^{2}\right.$ $\left(\mathbf{R}_{+}^{n+1}\right)$ ) and that $u(t)$ is a solution of (3.7). Therefore it is sufficient to prove that $u(t)$ is in the pre-domain $\mathrm{D}_{\mathrm{L}}$ and $\frac{\partial}{\partial y_{j}} u(t)(j=1, \ldots, n)$ in $L_{\mu}^{2}\left((0, \infty) ; L^{2}\left(\mathbf{R}_{+}^{n+1}\right)\right)$.

Since $g$ is in $\mathscr{D}\left(\mathbf{R}_{+}^{n+1}\right)$, from the corollary of Lemma 2.6 it is seen that $v(\tau)=(\tau-L)^{-1} g$ is in the pre-domain $\mathrm{D}_{\mathrm{L}}$, and further noting that $L g$ is also in $\mathscr{D}\left(\mathbb{R}_{+}^{n+1}\right)$, we can see that $L v(\tau)=(\tau-L)^{-1} L g$ is in the pre-domain $\mathrm{D}_{\mathrm{L}}$ and from the corollary of Lemma 2.7 we obtain the estimate

$$
\int_{-\infty}^{\infty}\|L v(\tau)\|_{s}^{2} d \sigma \leqq c(\mu, s)\|L g\|_{s}^{2}
$$

for any positive integer $s$ and $\mu=\operatorname{Re} \tau>\beta$. From the expression (2.16) anb (3.39) we can derive, by Schwarz's inequality,

$$
\left\|\left.u(t)\right|_{i s} ^{2} \leqq\right\| g\left\|_{s}^{2}+c(\mu, s) e^{2 \mu t}\right\| L g \|_{s}^{2}
$$

for any positive integer $s$ and for $\mu>\beta$. Hence by Sobolev's lemma we can see that $u(t)$ is a $C^{\infty}$-function of $(x, y)$ in $\overline{\mathbf{R}_{+}^{n+1}}$, and also $u(t)$ is in the pre-domain $\mathrm{D}_{\mathrm{L}}$. It is clearly seen that $\frac{\partial}{\partial y_{j}} u(t)(j=, \ldots, n)$ are in $L_{\mu_{0}}^{2}\left((0, \infty) ; L^{2}\left(\mathbf{R}_{+}^{n+1}\right)\right)$ for $\mu_{0}>\mu$. Thus the proof of Lemma 2.8 is complete. 


\section{$\S 4$. Existence Theorem}

In this section we shall prove Theorem 1.2. To do so we use HilleYosida thorem.

Theorem 4.1 (Hillo-Yosida). Suppose that the operator $L$ satisfies (1.2), then there exists a unique semi group $T_{t}$ having $L$ as the infinitesimal gencrator.

Remark. It is casily seen that the semi group $T_{t}$ assured by Theorem 4.1 can be identified with $S_{t}$ defined in the section 2 .

Applying Theorem 4.1 to the following equation

$$
\left\{\begin{array}{l}
\frac{d}{d t} u(t)=L[u(t)]+f(t) \\
u(0)=g
\end{array}\right.
$$

we have

Theorem 4.2. Suppose (1.2). Then for given $f(t)$ such that $f(t)$ and $L f(t)$ are in $\mathscr{E}_{t}^{0}\left(L^{2}\left(\mathbf{R}_{+}^{n+1}\right)\right)$ and for initial value $g$ in $\mathscr{D}(L)$, the unique solution of the equation (4.1) in $\mathscr{E}_{t}^{1}\left(L^{2}\left(\mathbf{R}_{+}^{n+1}\right)\right)$ and $\mathscr{D}(L)$ exists and is given by

$$
u(t)=T_{t} g+\int_{0}^{t} T_{t-s} f(s) d s .
$$

Moreover the following energy inequality holds

$$
\|u(t)\| \leqq c \cdot e^{\mu_{0} t}\|g\|_{1}+c \int_{0}^{t} e^{\mu_{0}(t-s)}\|f(s)\| d s
$$

By the above theorem we obtained the solution belonging to $\mathscr{E}_{t}^{1}\left(L^{2}\right.$ $\left(\mathbf{R}_{+}^{n+1}\right)$ ) and $\mathscr{D}(L)$ under the restriction such that $f(t)$ is in $\mathscr{E}_{t}^{0}(\mathscr{D}(L))$ and $g$ in $\mathscr{D}(L)$. Moreover, when $g$ is in $\mathscr{D}(L) \cap H^{1}\left(\mathbf{R}_{+}^{n+1}\right)$ and $f(t), f^{\prime}(t)$ and $\frac{\partial}{\partial y_{j}} f(t)(j=1, \ldots, n)$ are in $\mathscr{E}_{t}^{0}\left(L^{2}\left(\mathbf{R}_{+}^{n+1}\right)\right)$, we can obtain the solution in $\mathscr{D}(L) \cap \mathscr{E}_{t}^{1}\left(L^{2}\left(\mathbf{R}_{+}^{n+1}\right)\right) \cap \mathscr{E}_{t}^{0}\left(H^{1}\left(\mathbf{R}_{+}^{n+1}\right)\right)$.

Suppose that $f(t)$ is in $\mathscr{E}_{t}^{1}\left(L^{2}\left(\mathbf{R}_{+}^{n+1}\right)\right)$. Then the differentiation of the expression (4.2) with respect to $t$ leads us to 


$$
u^{\prime}(t)=T_{t}(L g+f(0))+\int_{0}^{t} T_{t-s} f^{\prime}(s) d s
$$

which gives

$$
\left\|u^{\prime}(t)\right\| \leqq c e^{\mu_{0} t}\left\{\|L g+f(0)\|+\int_{0}^{t}\left\|f^{\prime}(s)\right\| d s\right\} .
$$

We can remove the restriction in the argument of Theorem 4.2 such that $f(t)$ in $\mathscr{D}(L)$ and $L f(t)$ in $\mathscr{E}_{t}^{0}\left(L^{2}\left(\mathbf{R}_{+}^{n+1}\right)\right)$, using the estimate (4.4), that is

Lemma 4.1. If $f(t)$ is $\mathscr{E}_{t}^{1}\left(L^{2}\left(\mathbf{R}_{+}^{n+1}\right)\right)$ and $g$ in $\mathscr{D}(L)$, then there exists a unique solution of (4.1) belonging to $\mathscr{E}_{t}^{1}\left(L^{2}\left(\mathbf{R}_{+}^{n+1}\right)\right)$ and $\mathscr{D}(L)$, and satisfies (4.4).

Proof. For given $f(t) \in \mathscr{E}_{t}^{\mathscr{1}}\left(L^{2}\left(\mathbf{R}_{+}^{n+1}\right)\right.$, we can choose the sequence of functions $\left\{f_{n}(t)\right\}$ such that

1) $f_{n}(t)$ and $L f_{n}(t)$ are in $\mathscr{E}_{t}^{0}\left(L^{2}\left(\mathbf{R}_{+}^{n+1}\right)\right)$

2) $\left\|f_{n}(0)-f(0)\right\|$ and $\int_{0}^{t}\left\|f^{\prime}{ }_{n}(s)-f^{\prime}(s)\right\| d s$ tend to zero when $n \rightarrow \infty$.

Let $u_{n}(t)$ be the solution of (4.1) for data $\left(f_{n}(t), g\right)$. Then from (4.3) and (4.4) we have

$$
\begin{aligned}
& \max _{0 \leqq s \leqq t}\left\|u_{n}(s)-u_{m}(s)\right\|+\max _{0 \leqq s \leqq t}\left\|u_{n}^{\prime}(s)-u_{m}^{\prime}(s)\right\| \\
& \leqq c e^{\mu_{0} t}\left\{\left\|f_{n}(0)-f_{m}(0)\right\|+\int_{0}^{t}\left\|f_{n}^{\prime}(s)-f_{m}^{\prime}(s)\right\|_{1} d s\right\} .
\end{aligned}
$$

Therefore $\left\{u_{n}(t)\right\}$ converges and the limit $u(t)$ is in $\mathscr{E}_{t}^{1}\left(L^{2}\left(\mathbf{R}_{+}^{n+1}\right)\right)$ and satisfies (4.1) and (4.4). It follows clearly from (4.3) that the solution which we have constructed is the unique solution of (4.1).

Proof of Theorem 1.2. Let $u(t)$ be a solution obtained by Theorem 4.2. Put

$$
u_{\varepsilon}(t)=\rho_{\varepsilon} * u(t) .
$$

It is obvious that $\frac{\partial}{\partial y_{j}} u_{\varepsilon}(t)(j=1, \ldots, n)$ are in $\mathscr{E}_{t}^{1}\left(L^{2}\left(\mathbf{R}_{+}^{n+1}\right)\right) \cap \mathscr{E}_{t}^{0}\left(H^{1}\left(\mathbf{R}_{+}^{n+1}\right)\right)$ 
$\cap \mathscr{D}(L)$ and satisfy

$$
\left\{\begin{array}{l}
\frac{\partial}{\partial t}\left(\frac{\partial}{\partial y_{j}} u_{\varepsilon}(t)\right)=L\left(\frac{\partial}{\partial y_{j}} u_{\varepsilon}(t)\right)+\frac{\partial}{\partial y_{j}}\left\{\left[C, \rho_{\varepsilon}\right] u(t)+f_{\varepsilon}(t)\right\} \\
\frac{\partial}{\partial y_{j}} u_{\varepsilon}(0)=\frac{\partial}{\partial y_{j}} g_{\varepsilon}
\end{array}\right.
$$

where $f_{\varepsilon}(t)=\rho_{\varepsilon} * f(t), g_{\varepsilon}=\rho_{\varepsilon} * g$. Hence from (4.3) it follows

$$
\begin{aligned}
& \left\|\frac{\partial}{\partial y_{j}} u_{\varepsilon}(t)\right\| \\
& \leqq c e^{\mu_{0} t}\left\{\left\|\frac{\partial}{\partial y_{j}} g_{\varepsilon}\right\|+\int_{0}^{t}\left\|\left[C, \rho_{\varepsilon} \frac{\partial}{\partial y_{j}}\right] u(t)+\frac{\partial}{\partial y_{j}} f_{\varepsilon}(t)\right\| d t\right\} .
\end{aligned}
$$

Hence $\left\{\frac{\partial}{\partial y_{j}} u_{\varepsilon}(t)\right\}$ is Cauchy sequence in $\mathscr{E}_{t}^{0}\left(L^{2}\left(\mathbf{R}_{+}^{n+1}\right)\right)$ and it's limit is equal to $\frac{\partial}{\partial y_{j}} u(t)$. Limiting $\varepsilon \rightarrow 0$ in (4.5), we obtain

$$
\left\|\frac{\partial}{\partial y_{j}} u(t)\right\| \leqq c e^{\mu_{0} t}\left\{\|g\|_{1}+\int_{0}^{t}\left(\|u(t)\|+\left\|\frac{\partial}{\partial y_{j}} f(t)\right\|\right) d t\right\}
$$

for $j=1,2, \ldots, n$. The fact that $u(t)$ is a solution of (4.1) and (4.4) imply (1.3). This completes the proof of Theorem 1.2.

\section{References}

[1] Agmon, S., Problèmes mixtes pour les équations hyperboliques d'ordre supérieur, Colloque Internationaux du C.N.R.S. (1962), 13-18.

[2] Asano, K. and T. Shirota, On certain mixed problem for hyperbolic equations of higher order, Proc. Japan Acad. 45 (3), (1969), 145-148.

[3] Ikawa, M., Mixed problems for hyperbolic equations of second order, J. Math. Soc. Japan, 20 (1968), 580-608.

[4] Friedrichs, K. O., Pseudo-Differential Operator, Lecture note in Courant Institute, (1967-68).

[5] Friedrichs, K.O. and P.D. Lax, Boundary value problems for first order operators, Comm. Pure Appl. Math. 18 (1965), 355-388.

[6] Hersh, R., Mixed problems in several variables, J. Math. Mech. 12 (1963), 317334.

[7] - Boundary conditions for equations of evolution, Arch. Rational Mech. Anal. 16 (1964), 243-264.

[8] Kajitani, K., Mixed Problems for First Order Hyperbolic Systems, Master these to Kyoto University, 1969.

[9] Kreiss, O.K., Initial boundary value problems for hyperbolic systems, to appear. 
[10] Lax, P.D. and R.S. Phillips, Local boundary conditions for dissipative symmetric linear differential operators, Comm. Pure Appl. Math. 13 (1960), 427-455.

[11] Matsumura, M., Comportement des solutions de quelques problèmes mixtes pour certains systèmes hyperboliques symétriques à coefficients constants, Publ. RIMS Kyoto Univ. 4 (1968), 309-359.

[12] Mizohata, S., Theory of Partial Differential Equations, Iwanami, Tokyo, 1956 (Japanese).

[13] Mizohata, S., Quelques Problemes au Bord, du Type Mixte, pour des Equations Hyperboliques, Collège de France, (1966-67), 23-60.

[14] Miyatake, S., A remark on singular integral operators and reflection principle for some mixed problems, Proc. Japan Acad. 45 (1969), 547-551.

[15] Sadamatsu, T., On mixed problems for first order hyperbolic systems with constant coefficients, Proc. Japan Acad. 45 (1969), 686-691.

[16] Sarason, L., On hyperbolic mixed problems, Arch. Rational Mech. Anal. 16 (1964), 243-264.

[17] Strang, G., Hyperbolic initial boundary value problems in two unknowns, $J$. Differential Equations 6 (1969), 161-171. 